\title{
On Very Weak Positive Solutions to Some Semilinear Elliptic Problems With Simultaneous Singular Nonlinear and Spatial Dependence Terms
}

\author{
J.I. Díaz, J. Hernández and J.M. Rakotoson
}

\begin{abstract}
We use recent results by Díaz and Rakotoson concerning very weak solutions to linear boundary value problems in order to improve previous work on existence and properties of weak positive solutions to a model example of semilinear singular elliptic problem.
\end{abstract}

Mathematics Subject Classification (2010). Primary 35J60; Secondary 35J65.

Keywords. Nonlinear singular elliptic equations, positive very weak solutions.

\section{Introduction}

Semilinear elliptic equations have been studied in the last fifty years as models for many different phenomena arising in applications (population dynamics, combustion, chemical reactions, etc.). A typical example of reaction-diffusion equation of this type is

$$
\begin{cases}-\Delta u=f(x, u) & \text { in } \Omega, \\ u=0 & \text { on } \partial \Omega,\end{cases}
$$

where $\Omega$ is a bounded domain in $\mathbb{R}^{N}$, and $f$ is a smooth nonlinearity satisfying $f(x, 0) \geq 0$ for all $x$. But the situation where $f$ is not defined at the origin and goes to $+\infty$ when $u>0$ tends to 0 is also interesting for some applications and has been considered in a series of papers starting with the important work by Crandall, Rabinowitz and Tartar [7] (see also Stuart [22]) in the late seventies. A model problem for these singular nonlinearities is the following:

$$
P(a ; h) \begin{cases}-\Delta u=\frac{h(x)}{u^{a}} & \text { in } \Omega, \\ u=0 & \text { on } \partial \Omega,\end{cases}
$$


with $h(x) \geq 0$ on $\Omega$ and $a>0$ (some of our results remain valid for some $a<0$ but we shall not present them here). We send the reader to the next section for a collection of comments on other previous results in the literature.

This paper is a short presentation of a part of a longer work ([9]) in which we consider a larger class of semilinear singular problems including problem (1.2) as a special case. Here we shall limit ourselves mainly to the case in which,

$$
h(x)=\frac{g(x)}{d(x)^{b}}
$$

with $g \in L^{\infty}(\Omega)$ such that

$$
0<C_{g} \leq g(x)
$$

so that the problem becomes

$$
P(a, b ; g) \begin{cases}-\Delta u=\frac{g(x)}{d(x)^{b} u^{a}} & \text { in } \Omega, \\ u=0 & \text { on } \partial \Omega .\end{cases}
$$

Other different frameworks (case of general second order elliptic operators, more general singular source terms $f(x, u)$, the case in which the source term contains also an absorption possibly singular term as, e.g. $f(x, u)=\frac{h(x)}{u^{a}}-\frac{k(x)}{u^{\alpha}}$, etc.) are considered in [9]).

Our main goal is to prove some results on the existence of the so called (positive) very weak solutions for problem $P(a, b ; g)$ in the sense that we ask for a function $u \in L^{1}(\Omega)$, with $u(x)>0$ for a.e. $x \in \Omega$, such that $d^{-b} u^{-a} \in L^{1}(\Omega, d)$ (where $d(x)=\operatorname{dist}(x, \partial \Omega)$ and $L^{1}(\Omega, d)$ is the usual Lebesgue space weighted by the distance function $d(x))$ satisfying that

$$
-\int_{\Omega} u \Delta \varphi \mathrm{d} x=\int_{\Omega} \frac{g(x)}{d^{b} u^{a}} \varphi(x) \mathrm{d} x \text { for any } \varphi \in C^{2}(\bar{\Omega}), \varphi=0 \text { on } \partial \Omega .
$$

Note that, since every test function $\varphi \in C^{2}(\bar{\Omega})$ with $\varphi=0$ on $\partial \Omega$ must satisfy that $|\varphi(x)| \leq C d(x)$ for any $x \in \Omega$, and for some $C>0$, the above notion is well defined. Note also that this notion of solution is considerably weaker than the notions used by previous authors in the literature (see Section 2). The use of this type of very weak solutions (although for nonsingular semilinear problems) was started with the work by Haïm Brezis [1] (see also [2] and [12]) as an improvement of the usual $L^{1}$-weak type solutions (see [3]). This is one of the reasons why we are able to improve former results on the existence of solutions. We shall prove:

Theorem 1. Let $a+b>1$ with $b \in[0,2)$. Then there exists a very weak solution $u$ of $P(a, b ; g)$. Moreover $u \in C(\bar{\Omega}) \cap W_{\text {loc }}^{2, p}(\Omega)$ for any $p \in[1,+\infty)$.

Theorem 2. Let $a+b<1$. Then there exists a very weak solution $u$ of $P(a, b ; g)$. Moreover $u \in W_{0}^{1}\left(\Omega,|\cdot|_{N(\gamma), \infty}\right) \cap W_{\text {loc }}^{2, p}(\Omega)$, for any $\left.\gamma \in\right] 0,1[$ and for any $p \in[1,+\infty)$.

We point out that if $b \geq 2$ no very weak solution of $P(a, b ; g)$ may exist (see [13] and [9]) and that the uniqueness result of Crandall, Rabinowitz and Tartar [7] can be easily adapted to our framework (see [9]). Here we shall prove also that, after 
extending the notion of very weak solution of (1.2) in an obvious way to the case of a general coefficient $h(x)$, the above boundedness assumption on $g(x)$ can be removed in some cases:

Theorem 3. Let $a>0$ and let $h$ be such that there exist $C_{h}>0$ and $\gamma \in[0,1[$ such that $h(x) \geq C_{h}$ a.e. $x \in \Omega$ and $h \in L^{1}\left(\Omega, d^{\gamma-\frac{2 a}{1+a}}\right)$. Then there exists a very weak solution of $(1.2)$. Moreover $u \in W_{0}^{1}\left(\Omega,|\cdot|_{N(\gamma), \infty}\right)$ with $N(\gamma)=\frac{N}{N-1+\gamma}$.

We also give some results concerning the behavior of solutions near the boundary $\partial \Omega$ (see Theorems 4 and 5). Finally, as mentioned before, we send the reader to [9] for many other different results (specially concerning the case in which the equation involves some nonlinear terms of absorption type as, for instance, the ones previously considered in [10] and in many other papers).

\section{Previous work}

We start by recalling, more precisely, that it was proved in [7] that if $h(x)$ is in $C^{1}(\bar{\Omega})$ and $h(x)>0$ on $\bar{\Omega}$, there is a unique classical solution $u \in C^{2}(\Omega) \cap C(\bar{\Omega})$, with $u>0$ to (1.2), for any $a>0$. The proof is obtained by approximating problem (1.2) by regular nonlinearities where the classical method of sub and supersolutions was used to get existence of a unique solution to the approximate problem and then going to the limit to get a classical solution to the original singular problem. In [7] one can also find more information concerning regularity and boundary behavior of solutions. Namely: i) If $a>1$ solutions are Hölder continuous with exponent $2 /(1+a)$ and estimates for its boundary behavior are obtained by using rather involved ODE arguments in the "autonomous" case; ii) $u$ is Lipschitz up to the boundary $\partial \Omega$ if and only if $0<a<1$; iii) they give some additional information for the "borderline" case $a=1$.

Similar results on the existence of solutions were obtained by Stuart in [22] using, this time, an approximation argument with respect to the boundary condition. Actually both papers provide results for more general differential operators with smooth coefficients, not necessarily in divergence form, and for non-monotone nonlinearities as well. In that cases the approximate problems are more complicated and, indeed, a more sophisticated argument involving global bifurcation was used in [7]. Solutions with less regularity, namely in $W_{l o c}^{2, q}(\Omega) \cap C(\bar{\Omega})$, were obtained in [7] too, when less smoothness was assumed on the coefficients.

The same model problem (1.2) was considered again by Lazer and McKenna [19], this time with $h>0$ on $\bar{\Omega}$ and $h$ in $C^{\gamma}(\bar{\Omega})$ with $0<\gamma<1$. First, they greatly simplify the study of the boundary behavior by employing sub and supersolutions instead of ODE methods. On the other side, they give an interesting result showing that classical solutions are in the Sobolev space $H_{0}^{1}(\Omega)$ if and only if $0<a<3$. 
(This result was extended later by Zhang and Cheng [23] for $h(x)=d(x)^{\beta}$, with $\beta>0$, where $d(x)=\operatorname{dist}(x, \partial \Omega))$. They also provide a very simple proof of the fact that $\mathrm{u}$ is not in $C^{1}(\bar{\Omega})$ if $a \geq 1$.

The conditions on $h(x)$ were somewhat weakened by del Pino in [8], where $h$ is only assumed to be bounded, non-negative, and such that $h>0$ on some subset of positive measure of $\Omega$. Under these assumptions he proves, for any $a>0$, the existence of a positive solution in $C^{1, \gamma}(\Omega) \cap C(\bar{\Omega})$ for any $0<\gamma<1$, this time by using variational methods involving approximation for both $h(x)$ and the nonlinearity. The boundedness of the gradient of $u$ is obtained under an assumption which generalizes $a-\beta<1$ in the case of $h(x)=d(x)^{\beta}$. This last condition was used by Gomes [14] to show existence of a positive solution in $C^{1}(\bar{\Omega})$.

Another way to weakening the conditions on $h(x)$ was developed by Coclite in [4], [5], [6] by working in the Marcinkiewicz spaces $M^{r, p}(\Omega)$, where $r>N, p>$ 2. These spaces are such that $L^{\infty}(\Omega) \subset M^{r, p}(\Omega) \subset L^{1}(\Omega)$ and on the other side $M^{r, p}(\Omega) \nsubseteq L^{q}(\Omega)$ if $q>1$. (For example $d(x)^{-t} \in M^{r, p}(\Omega)$ for $0<t<1$ ). If $h(x) d(x)^{-a} \in M^{r, p}(\Omega)$, then he proves that there is a solution $u \in W_{l o c}^{2, q}(\Omega) \cap C(\Omega)$.

General regularity results were obtained by Gui and Hua Lin in [15] again for $h(x)=d(x)^{\beta}$, also with $\beta>0$. They prove that: i) if $a-\beta<1, u$ is in $C^{1,1+\beta-a}(\bar{\Omega})$, ii) if $a-\beta>1, u$ is in $C^{(2+\beta) /(1+a)}(\bar{\Omega})$, and iii) if $a+\beta=1$, then $u$ is in $C^{\gamma}(\bar{\Omega})$ for any $\gamma \in(0,1)$ but not in $C^{1}(\bar{\Omega})$. Similar results are obtained for some more general $h$ 's as well. Here the authors use again comparison methods in order to study the boundary behavior and regularity of solutions. This raises the question of the extension of this kind of results for suitable $\beta=-b$ with $b>0$.

A class of functions $h(x)$, which they call a Kato class, was introduced by Maagli and Zribi in [20]. This Kato class $K_{d}(\Omega)$ is such that $L^{p}(\Omega) \subset K_{d}(\Omega) \subset L^{1}(\Omega)$ for $p>N / 2$. If $h \in K_{d}(\Omega)$ and moreover it is locally Hölder continuous, then there exists a solution $u \in C^{2}(\Omega) \cap C(\bar{\Omega})$ to (1.2). A typical example of function $h \in K_{d}(\Omega)$ is $d(x)^{-b}$ with $b \in(0,1)$. Some time later they introduced in [21] a new Kato class $K(\Omega)$ of functions satisfying $K_{d}(\Omega) \subset K(\Omega) \subset L^{1}(\Omega, d)$. It is possible to show that $K(\Omega)$ is not contained in $L^{1}(\Omega)$. Now $h(x)=d(x)^{-b} \in K(\Omega)$ if $b \in(0,2)$, and hence it is in $K(\Omega)-K_{d}(\Omega)$ for $b \in[1,2)$. The same existence result is then proved under this more general assumption.

A partial existence result was also found in [17], [16], [18] where, as a particular case of more general results, it was proved directly that the same regularity holds for $b>0$ such that $a+b<1$, but only for solutions obtained by the method of sub and supersolutions there, and not for any solution. On the other hand, this is shown for general second order differential operators not necessarily in divergence form, and for more complicated singular nonlinearities as well.

Concerning non-existence results, it was shown by Ghergu in [13] that for $a>0$ and $b>0$ such that $b \geq 2$, there is no positive solution to $(1.2)$ in $C^{2}(\Omega) \cap C(\bar{\Omega})$. Some previous results concerning the case of (nonsingular) perturbation terms of absorption type can be found in [12]. 


\section{Proof of Theorems 1 and 2}

In this section we shall consider the case in which

$$
h(x)=\frac{g(x)}{d(x)^{b}}
$$

with $g \in L^{\infty}(\Omega)$ such that $0<C_{g} \leq g(x)$. We recall that the notion of (positive) very weak solution for this problem is the following: $u \in L^{1}(\Omega), u(x)>0$ for a.e. $x \in \Omega, d^{-b} u^{-a} \in L^{1}(\Omega, d)$ and

$$
\left\{\begin{array}{l}
-\int_{\Omega} u \Delta \varphi \mathrm{d} x=\int_{\Omega} \frac{g(x)}{d^{b} u^{a}} \varphi(x) \mathrm{d} x \\
\forall \varphi \in C^{2}(\bar{\Omega}), \varphi=0 \text { on } \partial \Omega .
\end{array}\right.
$$

We recall that the differentiability of the very weak solution of (1.1) was studied in Diaz and Rakotoson [11]. There, if $f(x, u)=f(x)$ with $f \in L^{1}\left(\Omega, \delta^{\alpha}\right), 0 \leq \alpha<1$, then $u$ satisfies

$$
u \in W^{1}\left(\Omega,|\cdot| \frac{N}{N-1+\alpha}, \infty\right),
$$

where the Sobolev-Lorentz spaces are defined by $W^{1}\left(\Omega,|\cdot|_{p, q}\right)=\left\{v \in W^{1,1}(\Omega)\right.$ : $\left.|\nabla v| \in L^{p, q}(\Omega)\right\}, 1 \leq p, q \leq+\infty$, with

$$
L^{p, q}(\Omega)=\left\{v: \Omega \rightarrow \mathbb{R} \text { measurable such that }|v|_{L^{p, q}}^{q}=\int_{0}^{|\Omega|}\left[t^{\frac{1}{p}}|v|_{* *}(t)\right]^{q} \frac{d t}{t}<+\infty\right\},
$$

where $|v|_{* *}(t)=\frac{1}{t} \int_{0}^{t}|v|_{*}(s) d s$, for $\left.t \in \Omega_{*}=\right] 0,|\Omega|\left[\right.$ and where $v_{*}$ denotes the decreasing rearrangement of $v$, defined by

$$
\left.v_{*}: \Omega_{*}=\right] 0,|\Omega|\left[\rightarrow \mathbb{R}, \quad v_{*}(s)=\inf \{t \in \mathbb{R}:|v>t| \leq s\} .\right.
$$

To prove Theorems 1 and 2 we shall need some auxiliary results. Let $\varphi_{1}>0$ be the first eigenfunction of the operator $-\Delta$ with homogeneous Dirichlet boundary conditions. Hence, $-\Delta \varphi_{1}=\lambda_{1} \varphi_{1}$ on $\Omega$ and we know that $\varphi_{1} \in H_{0}^{1}(\Omega) \cap W^{2, p}(\Omega), \forall p \in$ $[1,+\infty)$ and that there are two constants $c_{1}>0, c_{2}>0$ such that $c_{1} d(x) \leq \varphi_{1}(x) \leq$ $c_{2} d(x), \forall x \in \Omega$. We shall use later the following lemma.

Lemma 1. Let $t>0$. Then $-\Delta\left(\varphi_{1}^{t}\right)=\varphi_{1}^{t-2}\left[t(1-t)\left|\nabla \varphi_{1}\right|^{2}+\lambda_{1} t \varphi_{1}^{2}(x)\right]$. Moreover, if $0<t<1$, then there exists a constant $\eta>0$ such that

$$
H(x):=-\varphi_{1}^{2-t}(x) \Delta\left(\varphi_{1}^{t}(x)\right) \geq \eta>0 \text { for a.e. } x \in \bar{\Omega} .
$$

Proof. The first part comes from a straightforward computation. For the second part, we use that, by the Hopf maximum principle, there exists a neighborhood $\Omega_{0}$ of $\partial \Omega$, and a constant $\eta_{1}>0$ such that

$$
\left|\nabla \varphi_{1}(x)\right| \geq \eta_{1}>0, \forall x \in \Omega_{0}
$$

and that $\eta_{2}=\min _{\Omega \backslash \bar{\Omega}_{0}} \varphi_{1}>0$. Therefore, for $0<t<1$,

$$
H(x)=t(1-t)\left|\nabla \varphi_{1}\right|^{2}+\lambda_{1} t \varphi_{1}^{2}(x) \geq t \min \left(\eta_{1}^{2}, \eta_{2}^{2} \lambda_{1}\right)>0,
$$


and we can choose $\eta=t \min \left(\eta_{1}^{2}, \eta_{2}^{2} \lambda_{1}\right)$.

Now we shall study the existence of very weak solutions to $P(a, b ; g)$. Two different cases must be distinguished.

\subsection{Case $a+b>1$. Proof of Theorem 1}

Proof of Theorem 1. Let $0<\varepsilon<1, g_{\varepsilon} \in C(\bar{\Omega})$ such that $0<\frac{b_{0}}{2} \leq g_{\varepsilon}(x) \leq\|g\|_{\infty}$ and $g_{\varepsilon}(x) \rightarrow g(x)$ for a.a. $x \in \Omega$. Then there exists a function $u_{\varepsilon} \in H_{0}^{1}(\Omega) \cap W^{2, p}(\Omega)$, for all $1 \leq p<+\infty, u_{\varepsilon} \geq 0$ satisfying

$$
\left\{\begin{array}{cc}
-\Delta u_{\varepsilon}=\frac{g_{\varepsilon}(x)}{(d+\varepsilon)^{b}\left(u_{\varepsilon}+\varepsilon\right)^{a}} & \text { in } \Omega, \\
u_{\varepsilon}=0 & \text { on } \partial \Omega .
\end{array}\right.
$$

We need

Lemma 2. Let $\left.t_{1}=\frac{2-b}{1+a} \in\right] 0,1\left[\right.$. Then there exists a constant $c_{1}>0$ (large enough) such that if we set $w_{1}:=c_{1} \varphi_{1}^{t_{1}}$ then

$$
\left.-w_{1}^{a}(x) \Delta\left(w_{1}(x)\right) \geq \frac{g_{\varepsilon}(x)}{(d+\varepsilon)^{b}} \text { for a.e. } x \in \Omega, \forall \varepsilon \in\right] 0,1[\text {. }
$$

Moreover, for all $x \in \bar{\Omega}$, and for all $\varepsilon \in] 0,1\left[\right.$ we have $u_{\varepsilon}(x) \leq w_{1}(x)$.

Proof. One has for a. a. $x \in \Omega$

$$
\begin{aligned}
-w_{1}^{a}(x) \Delta\left(w_{1}(x)\right) & =c_{1}^{a+1} \varphi_{1}^{a t_{1}}(x) \varphi_{1}^{t_{1}-2}(x) H(x) \\
& =c_{1}^{a+1} \varphi_{1}(x)^{-b} H(x) \geq c_{1}^{a+1} \eta \varphi_{1}(x)^{-b} .
\end{aligned}
$$

Since there exists $c_{0}>0$ such that $\varphi_{1} \leq c_{0} d$, we obtain from the above relation that:

$$
-w_{1}^{a}(x) \Delta\left(w_{1}(x)\right) \geq c_{1}^{a+1} \eta c_{0}^{-b}(d(x)+\varepsilon)^{-b} .
$$

Let us choose $c_{1}>0$ such that

$$
c_{1}^{a+1} \eta c_{0}^{-b} \geq\|g\|_{\infty}
$$

Then from the two last relations, we have :

$$
-w_{1}^{a}(x) \Delta\left(w_{1}(x)\right) \geq\|g\|_{\infty}(d+\varepsilon)^{-b}(x) \geq \frac{g_{\varepsilon}(x)}{(d(x)+\varepsilon)^{b}} .
$$

Now, consider the case $a>0$, and suppose that there exists $x_{0} \in \bar{\Omega}$ such that $u_{\varepsilon}\left(x_{0}\right)>w_{1}\left(x_{0}\right)$. Then $x_{0} \in \Omega$ and we can assume that:

$$
\left(w_{1}-u_{\varepsilon}\right)\left(x_{0}\right)=\min _{x \in \Omega}\left(w_{1}-u_{\varepsilon}\right)(x)<0 .
$$

Thus we have that

$$
-\Delta\left(w_{1}-u_{\varepsilon}\right) \geq \frac{g_{\varepsilon}(x)}{(d(x)+\varepsilon)^{b}}\left[\frac{1}{w_{1}(x)^{a}}-\frac{1}{\left(u_{\varepsilon}+\varepsilon\right)^{a}(x)}\right] .
$$

for almost all $x \in \Omega$. By Bony's maximum principle, we have

$$
\liminf _{x \rightarrow x_{0}}\left(-\Delta\left(w_{1}-u_{\varepsilon}\right)\right)(x) \leq 0 .
$$


Thus from (3.7), (3.8) and (3.9), we deduce

$$
0<\frac{1}{w_{1}\left(x_{0}\right)^{a}}-\frac{1}{\left(u_{\varepsilon}\left(x_{0}\right)+\varepsilon\right)^{a}} \leq 0,
$$

which is absurd. A similar argument holds for the case $a \leq 0$.

Lemma 3. Let $t_{2}=\frac{2}{1+a}$. Then there exists a constant $c_{2}>0$ (small enough) such that if we set $w_{2}:=c_{2} \varphi_{1}^{t_{2}}$ then

$$
\left.-w_{2}^{a}(x) \Delta\left(w_{2}(x)\right) \leq \frac{g_{\varepsilon}(x)}{(d(x)+\varepsilon)^{b}}, \text { for a.e. } x \in \Omega, \forall \varepsilon \in\right] 0,1[\text {. }
$$

Moreover, for any $x \in \bar{\Omega}$ one has $\varepsilon+u_{\varepsilon}(x) \geq w_{2}(x)$.

Proof. One has

$$
-w_{2}^{a}(x) \Delta\left(w_{2}(x)\right)=c_{2}^{a+1} H(x) \leq\|H\|_{\infty} c_{2}{ }^{a+1} .
$$

for almost all $x \in \Omega$. We choose $c_{2}>0$ such that

$$
c_{2}^{a+1}\|H\|_{\infty} \frac{2}{b_{0}}\|d+1\|_{\infty}^{b}<1
$$

Thus from (3.10) and (3.11) we get

$$
-w_{2}^{a}(x) \Delta\left(w_{2}(x)\right) \leq \frac{C_{g}}{2} \frac{1}{\|(d+1)\|_{\infty}^{b}} \leq \frac{g_{\varepsilon}(x)}{(d(x)+\varepsilon)^{b}} .
$$

The rest of the proof is similar to the argument used in the proof of Lemma 2 and we drop it.

The proof of Theorem 1 ends with the following auxiliary result:

Lemma 4. There exists a function $u \in W_{l o c}^{2, p}(\Omega) \cap C(\bar{\Omega}), \forall p \in[1,+\infty[$ satisfying:

i) $w_{2}(x) \leq u(x) \leq w_{2}(x), \forall x \in \bar{\Omega}$.

ii) $-\Delta u(x)=\frac{\bar{g}(x)}{d(x)^{b} u(x)^{a}}$ in $D^{\prime}(\Omega)$.

iii) $u(x)=0$ for $x \in \partial \Omega$ (by extension).

Proof. Since we have for all $x \in \bar{\Omega}$

$$
w_{2}(x) \leq u_{\varepsilon}(x)+\varepsilon \leq w_{1}(x)+\varepsilon .
$$

we deduce that there exists a function $u \in L^{\infty}(\Omega)$ such that $u_{\varepsilon} \rightarrow u$ in $L^{\infty}(\Omega)$ weakly-star and we have

$$
w_{2}(x) \leq u(x) \leq w_{1}(x) \text { in } \Omega .
$$

Then, from the $W_{l o c}^{2, p}(\Omega)$-interior estimates, we have that for all $\Omega^{\prime} \subset \subset \Omega_{0} \subset \subset \Omega$ and for all $p \in\left[1,+\infty\left[\exists c_{p}>0\right.\right.$ such that

$$
\left\|u_{\varepsilon}\right\|_{W^{2, p}\left(\Omega^{\prime}\right)} \leq c_{p}\left[\left\|u_{\varepsilon}\right\|_{L^{\infty}(\Omega)}+\left\|\frac{g_{\varepsilon}(x)}{(d(x)+\varepsilon)^{b} u(x)^{a}}\right\|_{L^{p}\left(\Omega_{0}\right)}\right] .
$$

Hence

$$
\left\|u_{\varepsilon}\right\|_{W^{2, p}\left(\Omega^{\prime}\right)} \leq c_{p}\left(\Omega_{0}, w_{1}, w_{2}, g\right)<+\infty
$$


and it follows that $u \in C(\Omega) \cap W_{l o c}^{2, p}(\Omega), \forall p \in[1,+\infty)$. Since $\lim _{x \rightarrow \partial \Omega} w_{2}(x)=0=$ $\lim _{x \rightarrow \partial \Omega} w_{1}(x)$ we obtain $\lim _{x \rightarrow \partial \Omega} u(x)=0$. Therefore, $u \in C(\bar{\Omega})$ by extending $u$ by 0 at the boundary. We can easily pass to the limit in the equation satisfied by $u_{\varepsilon}$.

A similar argument can be used for $a \leq 0$.

\subsection{Case $a+b<1$. Proof of Theorem 2}

Lemma 5. There exists $c_{5}>0$ such that if $w_{5}=c_{5} \varphi_{1}$, we have, for a.e. $x \in \Omega$

$$
-c_{5}^{a} \varphi_{1}^{a} \Delta\left(c_{5} \varphi_{1}\right) \leq \frac{g_{\varepsilon}(x)}{(d(x)+\varepsilon)^{b}} .
$$

Moreover, if $u_{\varepsilon}(x)$ is defined as in the proof of Theorem 1 we have

$$
w_{5}(x) \leq u_{\varepsilon}(x)+\varepsilon \text { for any } x \in \bar{\Omega} .
$$

Proof. We have, for a.e. $x$

$$
\begin{aligned}
-(d+\varepsilon)^{b} c_{5}^{a} \varphi_{1}^{a} \Delta\left(c_{5} \varphi_{1}\right)(x) & \leq c_{5}^{a+1} \varphi_{1}^{a+1}(x)(d(x)+1)^{b} \\
& \leq c_{5}^{a+1}\|d+1\|_{\infty}^{b}\left\|\varphi_{1}\right\|_{\infty}^{a+1}
\end{aligned}
$$

we choose $c_{5}$ satisfying

$$
c_{5}^{a+1}\|d+1\|_{\infty}^{b}\left\|\varphi_{1}\right\|_{\infty}^{a+1}<\frac{C_{g}}{2} .
$$

Then we have the first inequality. The proof of (3.16) is the same as in Lemma 2 and we drop it.

Lemma 6. The function $h_{\varepsilon}:=\frac{g_{\varepsilon}(x)}{(d(x)+\varepsilon)^{b} u(x)^{a}}$ remains in a bounded set of $L^{1}\left(\Omega, d^{\gamma}\right)$ for all $\gamma \in(0,1)$.

Proof. From Lemma 5, we have, for all $\gamma>0$,

$$
\int_{\Omega}\left|h_{\varepsilon}(x)\right| d^{\gamma} \leq c \int_{\Omega} d^{\gamma-(b+a)} d x<+\infty,
$$

if $\gamma-(b+a)>-1$ that is, if $\gamma>b+a-1$. But $b+a-1 \leq 0<\gamma$, thus we have the result.

Corollary 1 . The sequence $\left(u_{\varepsilon}\right)_{\varepsilon>0}$ remains in a bounded set of $W_{0}^{1}\left(\Omega,|\cdot|_{N(\gamma), \infty}\right)$, $N(\gamma)=\frac{N}{N-1+\gamma}$ for all $\left.\gamma \in\right] 0,1[$.

Proof. It is enough to apply the regularity results of [11] to get that

$$
\left|\nabla u_{\varepsilon}\right|_{N(\gamma), \infty} \leq c\left|h_{\varepsilon}\right|_{L^{1}\left(\Omega, d^{\gamma}\right)} \leq c .
$$

End of the proof of Theorem 2. As a consequence of Corollary 1, we have shown the existence of a function $\left.u \in W_{0}^{1}(\Omega),|\cdot|_{N(\gamma), \infty}\right)$ such that $u_{\varepsilon} \rightarrow u$ and then strongly in $L^{1}(\Omega)$ and a.e. in $\Omega$. This allows us to pass to the limit in the equation. The last regularity result follows from standard bootstrap arguments. Moreover, we conclude that $u(x) \geq d(x) \forall x \in \bar{\Omega}$. 


\section{Behavior near the boundary and $\mathbf{H}_{0}^{1}$-regularity}

Theorem 4. There exist two positive constants $c_{1}$ and $c_{3}$ such that for any solution $u$ found in Theorem 1 we have that if $t:=\frac{2-b}{1+a}$ then

$$
c_{3} \varphi_{1}(x)^{t} \leq u(x) \leq c_{1} \varphi_{1}(x)^{t}, \text { for any } x \in \bar{\Omega} .
$$

From this theorem we shall derive:

Corollary 2. There exists a constant $c_{4}>0$ such that for all $\left.\gamma \in\right] \frac{a-1}{a+1} ; 1[$, we have

$$
|\nabla u|_{N(\gamma), \infty} \leq c_{4}\left|\frac{g}{d^{b+a\left(\frac{2-b}{1+a}\right)}}\right|_{L^{1}\left(\Omega, d^{\gamma}\right)}
$$

with $N(\gamma)=\frac{N}{N-1+\gamma}$.

Before passing to the proof of Theorem 4 we shall prove the following auxiliary result:

Lemma 7. There exists a constant $c_{3}>0$ such that if we define $w_{3}=c_{3} \varphi_{1}^{t_{1}}$ with $t_{1}=\frac{2-b}{1+a}$ then

$$
-w_{3}^{a}(x) \Delta\left(w_{3}(x)\right) \leq \frac{g(x)}{\varphi_{1}(x)^{b}}, \text { a.e. } x \in \Omega .
$$

Moreover, for any $x \in \bar{\Omega}$ we have $w_{3}(x) \leq u(x)$ whenever $u$ is a solution found in Theorem 1.

Proof. For any $x \in \Omega$ one has

$$
-w_{3}^{a}(x) \Delta\left(w_{3}(x)\right)=c_{3}^{a+1} \varphi_{1}(x)^{-b} H(x) \leq \frac{\|H\|_{\infty} c_{3}^{a}}{\varphi_{1}(x)^{b}} .
$$

we choose $c_{3}>0$ such that $\frac{C_{g}}{2} \geq\|H\|_{\infty} c_{3}^{a+1}$. Thus

$$
-w_{3}^{a}(x) \Delta\left(w_{3}(x)\right) \leq \frac{C_{g}}{2 \varphi_{1}(x)^{b}} \leq \frac{g(x)}{\varphi_{1}(x)^{b}} .
$$

Again, the proof of the inequality of the statement is similar to the one given in Lemma 2 and so we drop it.

End of the proof of Theorem 4. It follows from the above two Lemmas.

Proof of Corollary 2 (of Theorem 4). It will be a direct consequence of the two following auxiliary results.

Lemma 8. The function $h=\frac{g}{d^{b} u^{a}} \in L^{1}\left(\Omega, d^{\gamma}\right)$ for $\left.\gamma \in\right] \frac{b+a-1}{a+1}, 1[$, whenever $u$ is a solution found in Theorem 1.

Proof. We have

$$
\int_{\Omega}|h| d^{\gamma} d x \leq c\|g\|_{\infty} \int_{\Omega} d^{-b} d^{-a \frac{2-b}{1+a}} d^{\gamma} d x<+\infty
$$

for this choice of $\gamma$. 
Lemma 9. The function $v_{k} \in H_{0}^{1}(\Omega) \cap W^{2, p}(\Omega), \forall p \in\left[1,+\infty\left[,-\Delta v_{k}=h_{k}\right.\right.$, with $h_{k}:=T_{k}(h):=\min (h, k), k \geq 0$ satisfies:

i.) $\left|\nabla v_{k}\right|_{N(\gamma), \infty} \leq c(L, \Omega, \gamma)|h|_{L^{1}(\Omega, d \gamma)}$,

ii.) $0 \leq v_{k}(x) \leq v_{k+1}(x) \leq u(x), \quad \forall x \in \bar{\Omega}$,

iii.) $\left|v_{k}\right|_{W^{2, p}\left(\Omega^{\prime}\right)} \leq c_{p}\left(\Omega^{\prime}\right)<+\infty$ for all $\Omega^{\prime} \subset \subset \Omega$.

iv.) $v_{k}$ converges to $u$ everywhere in $\bar{\Omega}$.

Proof. Since $\left|h_{k}\right|_{L^{1}\left(\Omega, d^{\gamma}\right)} \leq|h|_{L^{1}\left(\Omega, d^{\gamma}\right)}<+\infty$ we deduce from [11] that $\left|\nabla v_{k}\right|_{N(\gamma, \infty)} \leq$ $c(L, \Omega, \gamma)|h|_{L^{1}\left(\Omega, d^{\gamma}\right)}$. Since $u$ and $v_{k}$ are in $W_{l o c}^{2, N}(\Omega) \cap C(\bar{\Omega})$ and we have $-\Delta\left(v_{k}-u\right) \leq$ 0 , by the maximum principle, we deduce that $0 \leq v_{k}(x) \leq u(x)$ for any $x \in \bar{\Omega}$. The same argument holds for $v_{k} \leq v_{k+1}$. Since $\left|h_{k}\right|_{L^{p}\left(\Omega_{0}\right)} \leq c\left(f, \Omega_{0}\right)<+\infty$, from the $W_{\text {loc }}^{2, p}(\Omega)$-regularity result, we have $\left\|v_{k}\right\|_{W^{2, p}\left(\Omega^{\prime}\right)} \leq c\left(p, \Omega^{\prime}, f\right)<+\infty$ for $\Omega^{\prime} \subset \subset \Omega$. Thus, there exists $w \in W_{l o c}^{2, p}(\Omega), \forall p \in[1,+\infty), w \in W_{0}^{1}\left(\Omega,|\cdot|_{N(\gamma), \infty}\right)$ such that $v_{k} \rightarrow w$, as $k \rightarrow+\infty$ and $0 \leq w(x) \leq u(x), \forall x \in \Omega$. Hence, $\lim _{x \rightarrow \partial \Omega} w(x)=0$ so that $w \in C(\bar{\Omega})$ and then we have

$$
\left\{\begin{array}{l}
-\Delta(w-u)=0 \\
w-u \in C(\bar{\Omega}) \cap W_{l o c}^{2, N}(\Omega) .
\end{array}\right.
$$

Thus by the maximum principle, we have $u=w \in W^{1}\left(\Omega,|\cdot|_{N(\gamma), \infty}\right)$.

A consequence of Theorem 4 is the following optimal $H_{0}^{1}$-regularity:

Theorem 5. Let $u$ be the solution given in Theorem 1 . Then $u \in H_{0}^{1}(\Omega)$ if and only if $a+2 b<3$.

Proof. Assume first that $u \in H_{0}^{1}(\Omega)$. Let us argue by contradiction to show that $a+2 b<3$. Otherwise, we shall have (using Theorem 1 )

$$
\int_{\Omega} h u \mathrm{~d} x=\int_{\Omega} \frac{f u}{d^{b} u^{a}} \mathrm{~d} x=+\infty .
$$

Using the sequence $v_{k}$ of Lemma 9 and taking $u$ as a test function, we shall have

$$
\int_{\Omega}\left|\nabla v_{k}(x)\right|^{2} \mathrm{~d} x=\int_{\Omega} h_{k} v_{k} \mathrm{~d} x \leq h_{k} u=\int_{\Omega} \nabla v_{k} \cdot \nabla u \mathrm{~d} x
$$

from which we derive that

$$
\int_{\Omega}\left|\nabla v_{k}\right|^{2} \mathrm{~d} x \leq c \int_{\Omega}|\nabla u(x)|^{2} \mathrm{~d} x<+\infty
$$

for some $c>0$. Therefore, $v_{k} \rightarrow u$ weakly in $H_{0}^{1}(\Omega)$. Thus, by Fatou's Lemma, one has from (4.1) that

$$
+\infty=\int_{\Omega} h u \mathrm{~d} x \leq \int_{\Omega}|\nabla u|^{2} \mathrm{~d} x<+\infty,
$$

which is a contradiction. Conversely, if $a+2 b<3$, then

$$
\int_{\Omega} h u \mathrm{~d} x=\int_{\Omega} \frac{f u}{d^{b} u^{a}} \mathrm{~d} x<+\infty .
$$


Therefore, since

we obtain

$$
\int_{\Omega} h_{k} v_{k} \mathrm{~d} x \leq \int_{\Omega} h u \mathrm{~d} x
$$

$$
\int_{\Omega}\left|\nabla v_{k}\right|^{2} \mathrm{~d} x \leq \int_{\Omega} h u \mathrm{~d} x<+\infty .
$$

Thus $v_{k}$ remains in a bounded set of $H_{0}^{1}(\Omega)$, from which we deduce that $u \in H_{0}^{1}(\Omega)$ (since $v_{k} \rightarrow u$ everywhere in $\bar{\Omega}$ ).

Remark. If $b<0$, the same argument can be done to recover several results given by [19] and [15] (see also [16]).

\section{Proof of Theorem 3}

Proof of Theorem 3. Let $h_{\varepsilon} \in L^{\infty}(\Omega), h_{\varepsilon} \rightarrow h$ a.e., $h_{\varepsilon}$ belongs to a bounded set of $L^{1}\left(\Omega, d^{\gamma-\frac{2 a}{1+a}}\right), h_{\varepsilon} \geq \frac{C_{h}}{2}$. Consider $u_{\varepsilon} \in H_{0}^{1}(\Omega) \cap W^{2, p}(\Omega), \forall 1 \leq p<+\infty$ satisfying $u_{\varepsilon} \geq 0$ and

$$
-\Delta u_{\varepsilon}=\frac{h_{\varepsilon}}{\left(u_{\varepsilon}+\varepsilon\right)^{a}} \text { in } \Omega .
$$

Let us choose $c>0$ (small enough) such that if $w=c \varphi_{1}^{t}$ with $t=\frac{2}{1+a}$, then $-w^{a} \Delta w \leq \frac{C_{h}}{2}$. Therefore, we have

$$
-\Delta w-\frac{h_{\varepsilon}}{w^{a}} \leq 0=-\Delta u_{\varepsilon}-\frac{h_{\varepsilon}}{\left(u_{\varepsilon}+\varepsilon\right)^{a}} .
$$

Then by the maximum principle, we deduce that $w \leq u_{\varepsilon}+\varepsilon$. Consequently, one obtains

$$
\int_{\Omega} \frac{\left|h_{\varepsilon}\right|}{\left(u_{\varepsilon}+\varepsilon\right)^{a}} d^{\gamma} d x \leq c \int_{\Omega} d^{\gamma} d^{-\frac{2 a}{1+a}} h_{\varepsilon} d x \leq C,
$$

for some $C>0$. From the results of [11], we deduce that $u_{\varepsilon}$ remains in a bounded set of $W_{0}^{1}\left(\Omega,|\cdot|_{N(\gamma), \infty}\right)$. Therefore there exists $u \in W_{0}^{1}\left(\Omega,|\cdot|_{N(\gamma), \infty}\right)$ such that $u_{\varepsilon} \rightarrow u$ weakly in $W_{0}^{1}\left(\Omega,|\cdot|_{N(\gamma), \infty}\right)$ and strongly in $L^{1}(\Omega)$. Thus, we can pass to the limit in $(5.1)$.

\section{References}

[1] H. Brezis, Une équation semi-linéaire avec conditions aux limites dans $L^{1}$, unpublished. Personal communication to J.I. Díaz.

[2] H. Brezis, T. Cazenave, Y. Martel and A. Raminandrisoa. Blow-up for $u_{t}-\Delta u=g(u)$ revisited. Adv. Differential Equations 1 (1996), 72-90.

[3] H. Brezis and W. Strauss. Semilinear Elliptic Equations in L ${ }^{1}$. J. Math. Soc. Japan, 25 (1973) 565-590.

[4] M. M. Coclite. On a singular nonlinear Dirichlet problem II. Bolletino Unione Mat. Ital. B 5 (1991), 955-975. 
[5] M.M. Coclite. On a singular nonlinear Dirichlet problem III. Nonlinear Anal. 21(1993), 547-564.

[6] M.M. Coclite. On a singular nonlinear Dirichlet problem IV. Nonlinear Anal. 23 (1994), 925-936.

[7] M.G.Crandall, P.H.Rabinowitz and L. Tartar. On a Dirichlet problem with singular nonlinearity. Comm. PDEs 2 (1977), 193-222.

[8] M. del Pino. A global estimate for the gradient in a singular elliptic boundary value problem. Proc. Roy. Soc. Edinburgh 122A (1992), 341-352.

[9] J.I.Díaz, J. Hernández and J.M.Rakotoson. On very weak positive solutions to some singular second order semilinear elliptic problems. In preparation.

[10] J.I.Díaz, J.M.Morel and L.Oswald. An elliptic equation with singular nonlinearity. Comm. PDEs 12 (1987), 1333-1344.

[11] J.I.Díaz and J.M.Rakotoson. On the differentiability of very weak solutions with righthand side data integrable with respect to the distance to the boundary, J. Funct. Anal. 357 (2009), 807-831.

[12] J. I. Díaz and J.M. Rakotoson. On very weak solutions of semilinear elliptic equations with right hand side data integrable with respect to the distance to the boundary, Discrete and Continuum Dynamical Systems, 27 (2010) 1037-1058.

[13] M. Ghergu, Lane-Emden systems with negative exponents, J. Functional Analysis 258 (2010), 3295-3318.

[14] S.N. Gomes. On a singular nonlinear elliptic problem. SIAM J. Math. Anal. 17 (1986), 1259-1269.

[15] C. Gui and F. Hua Lin. Regularity of an elliptic problem with singular nonlinearity. Proc. Roy. Soc. Edinburgh 123A (1993), 1021-1029.

[16] J.Hernández and F.Mancebo. Singular elliptic and parabolic equations. In Handbook of Differential equations (ed. M. Chipot and P. Quittner), vol. 3. Elsevier, 2006, 317-400.

[17] J. Hernández, F. Mancebo and J.M. Vega. On the linearization of some singular, nonlinear elliptic problems and applications. Annls. Inst. H. Poincaré, Analyse non Linéaire 19 (2002), 777-813.

[18] J.Hernández, F.Mancebo and J.M.Vega. Positive solutions for singular nonlinear elliptic equations. Proc. Roy. Soc. Edinburgh 137A (2007), 41-62.

[19] A.C. Lazer and P. J. McKenna. On a singular nonlinear elliptic boundary value problem. Proc. Amer. Math. Soc. 111 (1991), 721-730.

[20] H. Mâagli and M. Zbiri. Existence and estimates of solutions for singular nonlinear elliptic problems. J. Math. Anal. Appl. 263 (2001), 522-542.

[21] H. Mâagli and M. Zbiri. On a new Kato class and singular solutions of a nonlinear elliptic equation in bounded domains of $\mathrm{R}^{n}$. Positivity 9 (2005), 667-686.

[22] C.A. Stuart. Existence and approximation of solutions of nonlinear elliptic equations. Math. Z. 147 (1976), 53-63.

[23] Z. Zhang and J.Cheng. Existence and optimal estimates of solutions for singular nonlinear Dirichlet problems. Nonlinear Anal. 57 (2004), 473-484. 


\title{
Acknowledgment
}

The research of the first and second author was partially supported by the project ref. MTM200806208 of the DGISPI (Spain) and the Research Group MOMAT (Ref. 910480) supported by UCM and, in the case of the first author, also from the ITN "FIRST" of the Seventh Framework Programme of the European Community's (grant agreement number 238702 .

\author{
J.I. Díaz \\ Departamento de Matemática Aplicada \\ Universidad Complutense de Madrid \\ Plaza de las Ciencias No. 3 \\ 28040 Madrid \\ Spain \\ e-mail: diaz.racefyn@insde.es \\ J. Hernández \\ Departamento de Matemáticas \\ Universidad Autonóma de Madrid \\ 28049 Madrid \\ Spain \\ e-mail: jesus.hernandez@uam.es
}

J.M. Rakotoson

Laboratoire de Mathématiques et Applications

Université de Poitiers

Boulevard Marie et Pierre Curie, Téléport 2, BP 30179

86962 Futuroscope Chasseneuil Cedex

France

e-mail: rako@math.univ-poitiers.fr

Received: March 31, 2011. 